\title{
Twenty-Year Planning Study for the Relativistic Heavy Ion Collider Facility at Brookhaven National Laboratory
}

\author{
Prepared by \\ Brookhaven National Laboratory \\ with the \\ RHIC Scientific Community
}

For the

U.S. Department of Energy

Office of Nuclear Physics

December 31, 2003

Upton, New York 


\section{Table of Contents}

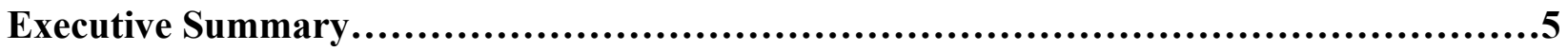

Section I Overview of the RHIC Planning Process...................................7

Section II The Five-Year Run Plan (FY 2004 - FY 2008)................................9

Section III Near-Term Upgrades for Detectors and Machines..............................14

Section IV The Mid-Term Outlook - RHIC II.......................................16

Section V The Long-Term Outlook - eRHIC..............................................19

\section{Appendices}

Appendix 1 RHIC Planning Group....................................................23

Appendix 2 Agenda for the Open Meeting on RHIC Planning, December 3-4, 2003......24

Appendix 3 Web References........................................................25

Appendix 4 Budget Table for FY 2004 - FY 2008..................................26

This document contains figures in color. The figures should be viewed in color. This work was performed under the auspices of the U.S. Department of Energy, Contract No. DE-AC02-98CH10886. 


\section{Executive Summary}

At the request of DOE's Office of Nuclear Physics (ONP), Brookhaven National Laboratory (BNL) has created this planning document to assemble and summarize a planning exercise that addresses the core scientific thrust of the Relativistic Heavy Ion Collider (RHIC) for the next twenty years and the facilities operation plan that will support this program. The planning work was carried out by BNL in close collaboration with the RHIC user community and within budgetary guidelines for the next five years supplied by the ONP. The resulting plans were reviewed by the BNL High Energy and Nuclear Physics Program Advisory Committee (PAC) at a special RHIC planning meeting held in December 2003.

Planning input from each of the four RHIC experimental collaborations was absolutely central to the preparation of this overall Laboratory plan. Each collaboration supplied two key documents, a five-year "Beam Use Proposal" and a ten-year "Decadal Plan". These plans are posted on the BNL website http://www.bnl.gov/henp/, along with other planning documents germane to this paper, such as the complete written reports from the August and December 2003 PAC meetings that considered the five-year and decadal planning documents of the four RHIC collaborations and offered advice and commentary on these plans. Only in these collaboration documents can the full physics impact of the RHIC program be seen and the full scope of the efforts put into this planning process be appreciated. For this reason, the maximum value of the present planning paper can only be realized by making frequent reference to the collaboration documents.

From this input, the Laboratory declares that our highest priority for the RHIC facility for the next five years is to meet the two Five-Year Critical Goals listed in Section II:

a) To follow up on the watershed results of the first RHIC runs by making definitive experimental statements on the existence of the quark gluon plasma and determining its essential properties.

b) To obtain spin-polarized p-p data samples of sufficient sensitivity to address the core physics questions of the RHIC spin program, including direct determination of the spindependent gluon structure functions.

It is also a high priority of the Laboratory to carry out essential R\&D for future improvements of the machine and detectors, and to implement certain key upgrades on a short time scale to maximize the physics output during this critical period of unchallenged discovery potential for the RHIC program.

BNL intends to pursue these priorities even in the Constant Effort budget scenario provided by ONP. We supply in Table 3 of this document, a budget plan that will meet this constraint and deliver 27 weeks of RHIC facility operations each year. A plan for an R\&D program is also incorporated in the Table 3 five-year funding plan and discussed in Section III.

The "Full Utilization" scenario for RHIC, on the other hand, corresponds to 37 weeks of operation annually, and was the basis for the first DOE Operations Review for RHIC in 1996. (It should also be noted that the Project Management Plan, approved by DOE as part of the "Key Decision 3" approval for RHIC construction in 1992, called for 38 weeks per year of operation as one of the technical performance objectives.) Full Utilization of RHIC remains Brookhaven's goal. In the course of this planning exercise, however, we discovered that a very modest $3 \%(\$ 4 \mathrm{M})$ increment in the constant-effort annual RHIC funding will increase the running to 32 weeks per year and, most importantly, result in as much as a 30\% gain in physics data taking time and, in 
some scenarios, nearly double the net physics output over a four-year sequence of runs. In fact, we concluded and indicate in this report, that 27 weeks per year is sub-critical for the type of running required for the RHIC program and 32 weeks is really the proper threshold level for a healthy program in both heavy ion and spin physics at RHIC.

In our longer-term planning for RHIC, BNL intends to pursue additional central questions of heavy ion and spin physics with the high-luminosity, "rare probes" capability of RHIC II. The RHIC II project, identified in DOE's plan, "Facilities for the Future of Science - a Twenty-Year Outlook” announced by DOE Secretary of Energy, Spencer Abraham, on November 10, 2003, will supply major upgrades of the RHIC collider and detectors thereby providing new capability for studying extremely rare processes with high precision in high-energy nucleus-nucleus, protonnucleus, and proton-proton collisions.

Following the RHIC II luminosity upgrade project, the DOE plan shows the addition of electron-ion collisions capability, provided by completion of the eRHIC project. The eRHIC project will provide an electron beam of energy up to $10 \mathrm{GeV}$ that collides with a high intensity beam of RHIC II in a new experimental detector, to extend the reach of RHIC physics to unexplored kinematic regimes and make fundamentally new physics processes accessible to this unique facility.

Our plan is to implement this capability in such a way as to allow an uninterrupted experimental program as the RHIC II upgrades are phased in, beginning near the end of the present decade and extending to a period 10-15 years from now, at which time the facility will be implementing the electron-beam capabilities of eRHIC.

The sequence of proposed RHIC upgrades is outlined in Sections IV and V of this plan. Preliminary cost estimates for RHIC II and eRHIC were presented to the NSAC sponsored "Future Facilities Workshop" held at Rutgers University on February 15, 2003. These estimates were provided in FY 2003 dollars, and included provisions for engineering, design, contingency, and BNL overhead. The white paper presented to NSAC is available at the above-noted BNL website.

When the BNL PAC completed their consideration of the plan summarized above, they provided a written report to the Laboratory with their advice and commentary. From this document, available on the website noted above (under HENP Program Advisory Committee), we quote here summary language that characterizes the PAC view of the scientific promise of the planned RHIC program and the outcome of the planning that supports this program:

"There seems little doubt that with continued and upgraded detection equipment and adequate running time a great deal of the deeper physics nature of this new state of matter [QGP] can be determined." and "We turn next to the laboratory plans for the next ten years. We strongly support the scientific targets of the upgrade plans. Each of them adds a capability that allows deeper insight into the nature of the quark gluon-plasma state and its production and evolution." and "While it is abundantly clear that a most interesting and important physics research program can be carried out within the constraints of this [27 weeks/year] guidance, it is also clear that very serious loss of physics knowledge will result if the program is constrained to this level for an extended period. To carry out a program under this constraint, major tradeoffs must be made and entire aspects of the program deleted."

We hope the plans presented in the subsequent sections of this paper, along with the summary comments of the BNL PAC, will convey the extraordinary value and outstanding science of the RHIC program and the credibility of its planned implementation under DOE provided budgetary constraints in a way that is helpful to the agency. We further hope that the modest funding increment suggested in this document will receive careful consideration by the agency planners. 


\section{Overview of the RHIC Planning Process}

This document summarizes the effort by Brookhaven National Laboratory (BNL), in close concert with the RHIC user community, to map the core scientific priorities for RHIC research onto a realistic schedule for facility operations and upgrades over the next two decades. The resulting scientific program was reviewed by the BNL High Energy and Nuclear Physics Program Advisory Committee (PAC) at a special RHIC planning meeting in December 2003, during which the PAC provided advice and commentary on the presented long-term RHIC program. The DOE's Office of Nuclear Physics (ONP) provided budgetary guidance for the next five years of RHIC facility operations and this guidance was fully incorporated into the planning process.

In the initial three yearly data runs to date, beginning with the first $\mathrm{Au} A u$ collisions in June 2000, RHIC has already provided an international community of more than 1000 scientists with colliding beams of heavy nuclei at energies up to $100 \mathrm{GeV} /$ nucleon, extending this energy frontier by an order of magnitude, and opening a new realm of research into the basic structure and origin of matter through direct studies of extended nuclear volumes under conditions that reveal the fundamental interactions of quantum Chromodynamics, the realm of the postulated quark gluon plasma.

During these inaugural runs, RHIC also achieved the first-ever colliding beams of spinpolarized protons, establishing a path to take the next steps in a world-wide effort to understand the spin structure of the nucleon at the level of its constituent quarks and gluons.

The first results from RHIC have opened a landscape of vast discovery potential, and in doing so have provided the community of users with a clear set of challenges in charting the nearterm course for utilizing this new facility - now the largest U.S. nuclear physics facility. Over the next eight to ten years this community will map out experimentally, the phenomena that bear on the existence and properties of the quark gluon plasma, while in the same time period, also bringing the machine and detectors to the highly refined level of performance required to make definitive new measurements with high-energy polarized proton collisions.

Over a longer term, extending out 20 years and more, the scientific questions and accelerator issues that will shape the Laboratory's plans for the evolution of the RHIC facility into the RHIC II and eRHIC eras have been further sharpened in light of the data already analyzed and the related theoretical advances. Our BNL plans for the evolution of the RHIC facility were strongly supported in the newly released DOE science planning document, "Facilities for the Future of Science $-a$ Twenty-Year Outlook" announced by Secretary of Energy, Spencer Abraham, on November 10, 2003.

In this planning document, these matters are addressed as follows:

Section II. The Five-Year Run Plan (2004 - 2008)

Section III. Near-Term Upgrades for Detectors and Machines

Section IV. The Mid-Term Outlook - RHIC II

Section V. The Long-Term Outlook - eRHIC

The basis for this document is a report prepared by the RHIC Planning Group, convened in August 2003 as a means of engaging the broad community of RHIC users, accelerator groups, and BNL management in a focused effort to integrate the ongoing work of several planning activities: 
- The Beam Use Proposals and Decadal Plans developed and provided by the RHIC experimental collaborations at the request of the Associate Laboratory Director for High Energy and Nuclear Physics

- Recommendations of BNL's HENP Program Advisory Committee and RHIC Detector Advisory Committee

- Requirements developed by BNL's Collider-Accelerator Department for continued improvement of machine performance

- DOE Office of Nuclear Physics budget guidance

- The NSAC Long Range Planning process

The membership of the Planning Group is shown in Appendix 1. It consisted of representatives from each of the experimental collaborations, the RHIC machine groups, the RHIC Computing Facility, the BNL nuclear theory group, and the principal scientific advisory committees. A draft version of the Planning Group report was publicly presented at the Open Meeting on RHIC Planning, December 3-4, 2003. This final report incorporates input from the discussion at that meeting. The agenda of the Open Meeting is shown in Appendix 2.

Finally, we supply a summary of the time sequence of the machine and detector upgrades that are discussed in Sections II-V of this paper:

Near Term (starts within the next 1-3 years):

- Electron Beam Ionization Source (EBIS)

$\$ 17.5 \mathrm{M}$

- $\quad$ STAR Time-of-Flight System (TOF)

$\$ 5 \mathrm{M}$

- PHENIX Silicon Tracker

$\$ 6 \mathrm{M}$

Additional smaller detector upgrades will be provided as R\&D and capital funding allow.

Mid-Term (starts within the next 5-10 years):

RHIC II:

- Electron Beam Cooling

- Major Detector Upgrades

- Total Estimated Cost

Long Term (starts within 10-15 years):

eRHIC:

- $10 \mathrm{GeV}$ electron ring

$\$ 326 \mathrm{M}$

- Detector and insertion region

- Total Estimated Cost
$\$ 53 \mathrm{M}$

$\$ 100 \mathrm{M}$

\$153M (FY 03 dollars)
$\$ 185 \mathrm{M}$

\$511M (FY 03 Dollars) 
II. The Five-Year Run Plan (2004 - 2008)

The Five-Year Critical Goals to be achieved during this period are:

a. To follow up on the watershed results of the first RHIC runs by making definitive experimental statements on the existence of the quark gluon plasma and determining its essential properties.

b. To obtain spin-polarized p-p data samples of sufficient sensitivity to address the core physics questions of the RHIC spin program, including direct determination of the spin-dependent gluon structure functions.

Each of the four RHIC experimental collaborations provided its own view of the five-year planning process through the Five-Year Beam Use Proposals submitted to BNL in August 2003, and presented to the PAC in September. The collaborations were asked to consider two cases: A "Constant Effort" funding scenario (based on the FY 2004 presidential budget), which was assumed to correspond to 27 weeks of cryogenic operations per year, and a "Full Utilization" scenario corresponding to 37 weeks of operation annually, as was initially proposed for RHIC in the original DOE facilities operations objectives.

Key accelerator performance guidance for determining the scientific productivity of a particular RHIC operations scenario is given in the document "RHIC Collider Projections (FY 2004 - FY 2008)", by T. Roser and W. Fischer in June 2003. (This document can be found on the web at www.bnl.gov/henp/). In broad outline, it provides the following constraints on the utilization of RHIC during a single cryogenic run:

1. Cool-down and warm-up of the RHIC magnet rings require a total of 3 weeks.

2. Each mode of running requires 2 weeks of machine set-up and 3 weeks of machine ramp-up to stable physics operation. (A change in energy, after stable operation is achieved with a particular beam species pair, requires only 2 weeks.)

3. Both minimum and maximum luminosity projections are given. The minimum values assume little improvement over presently-established performance. The maximum values assume steady improvement in performance, requiring long periods of sustained running in a given mode.

In this planning model the weekly integrated luminosity increases linearly in time, reaching the final target value for luminosity improvement only after 14 weeks of production running.

From the detailed Five-Year Beam Use Proposals - particularly those of STAR and PHENIX - it is evident that achieving the two Five-Year Critical Goals in a timely manner is a severe challenge in light of the constraints on beam availability imposed by the need to run in a number of beam species modes, along with the need for machine development to reach necessary integrated luminosity levels of performance.

The design target for full utilization of the RHIC facility calls for a 37 week/year operations schedule. To date, the amount of running has been limited by the RHIC operations budget. Under the present guidance from DOE, this budget is expected to remain relatively flat at the level of FY 2004 in constant dollars (ie., inflation corrected to first order). This "Constant Effort" budget scenario is estimated to support 27 weeks per year of running. 
The Beam Use Proposals from the experimental collaborations address both the Full Utilization scenario and the Constant Effort case. In the Full Utilization scenario, the collaborations have outlined proposed programs for the next five years that would address the Critical Goals by providing large samples of full-energy Au-Au collisions and adequate comparison runs with collisions of lighter ions and $\mathrm{Au}-\mathrm{Au}$ collisions over a range of energies, while at the same time providing the necessary machine development to ensure high-luminosity polarized proton running during this period.

The current guidance from DOE on future budget levels requires that we address the Constant Effort case though we have also studied more generous allocations of beam running time. The Constant Effort case received the most attention during our planning discussions, as its budget constraints imposed severe challenges that required difficult compromises.

From the presentations in the Five-Year Beam Use Proposals we derived a minimal set of physics runs and data samples to address the Five-Year Critical Goals listed at the beginning of this Section. We display this minimal set of beam species runs in Table 1.

\begin{tabular}{|c|l|}
\hline Heavy Ions & \multicolumn{1}{|c|}{ Physics Data Goals for Experiments } \\
\hline 1. & $\begin{array}{l}\text { A 200 GeV Au Au run }\left(>300 \mu \mathrm{b}^{-1}\right) \text { in } 2004 \text { to follow-up on high } \mathrm{p}_{\mathrm{T}} \text { results, } \\
\text { and get the first sizeable sample of } \mathrm{J} / \psi .\end{array}$ \\
\hline 2. & $\begin{array}{l}\text { Energy scan run: } \\
\text { Au Au at } 1 \text { or 2 lower energies. } 50-100 \mu \mathrm{b}^{-1} \text { total }\end{array}$ \\
\hline 3. & $\begin{array}{l}\text { Species scan run: } \\
1-2 \text { lighter ions at } 200 \mathrm{GeV} .3-6 \mathrm{nb}^{-1} \text { total }\end{array}$ \\
\hline 4. & $\begin{array}{l}\text { A long Au Au run at } 200 \mathrm{GeV} \text { in } 2007 \text { or } 2008 \text {, with upgraded detector } \\
\text { capability for open charm and particle i.d. at high } \mathrm{p}_{\mathrm{T}}\left(\geq 2000 \mu \mathrm{b}^{-1}\right)\end{array}$ \\
\hline Polarized Protons & $\begin{array}{l}15-20 \text { weeks of "development" in } 2004-2005 \text { (this would include physics } \\
\text { data, but is required primarily to get the luminosity and polarization up to } \\
\text { required levels). }\end{array}$ \\
\hline 1. & Full-capability spin data at $200 \mathrm{GeV.} \geq 150 \mathrm{pb}^{-1}$ \\
\hline 2. &
\end{tabular}

Table 1. Minimal running requirements for the period 2004 - 2008: Note that the sample sizes indicated here are for delivered integrated luminosities. The actual recorded data samples used in physics analysis will be smaller by factors of 2-3.

It must be noted that Table 1 omits several measurements that are strongly advocated by the experimental community. Omitted from this table are: a second, long deuteron-Au run, a serious omission in light of the recent BRAHMS results on forward hadron suppression in d-Au data; any significant start on $500 \mathrm{GeV}$ pp running (W production); and runs of sufficient length with lower energy $\mathrm{Au}-\mathrm{Au}$ collisions and lighter ion species to study rare probes. Although these omitted runs offer exciting and rewarding physics opportunities, they simply cannot be accommodated in the 27week minimal scenario.

The Five-Year Beam Use Proposals for the Constant Effort case are the result of a lengthy and intense effort by the experimental collaborations to make scientific choices and impose programmatic discipline and efficiencies to achieve the most physics possible with 27 running weeks per year. Nonetheless, it does not seem possible, even under optimistic projections for 
machine and detector performance, to reach all of the requirements in Table 1 within a 5-year, 27week/year plan. A clear conclusion, articulated strongly in both the PHENIX and STAR Five-Year Beam Use proposals, is that a running plan providing only 27 weeks per year does not satisfy the minimum requirements of the scientific program. This is not simply an incremental matter that can be resolved by allowing another year or so of running. It is a fundamental problem that arises from the fact that the overall scientific program requires large data samples under many different running conditions. This circumstance is combined with relatively long set-up times for each change in running conditions, and with the need for long sustained runs to achieve the necessary maturemachine performance improvements.

The RHIC Planning Group considered ways of improving the utilization of the facility under a constant-effort budget constraint by allowing runs to be combined across fiscal years to improve the flexibility for long runs, while reducing the number of warm-up/cool-down cycles over the fiveyear period. The group examined the following three scheduling variations, each scheme of which assumes that the FY 2004 run proceeds as presently scheduled:

Scheme 1: The four runs in FY 2005 - FY 2008 are combined to provide two 54-week runs, interspersed with 50-week shutdowns.

Scheme 2: The four runs in FY 2005 - FY 2008 are combined to provide three runs of 35 to 37 weeks each, with correspondingly shorter shut-down periods.

Scheme 3: Relatively short runs dedicated to spin running and development are interspersed with longer runs for heavy ions.

These schemes are illustrated in Figure 1.

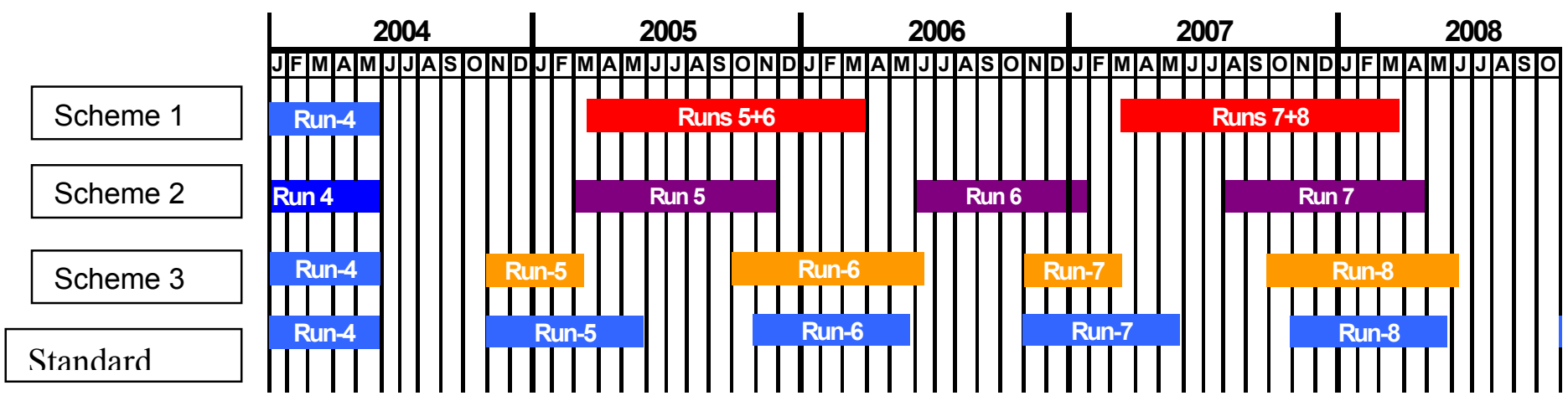

Figure 1. Alternate schemes for Constant-Effort run plans. The "standard" 27 weeks/yr plan is shown at the bottom.

There is general agreement that the extreme case represented by Scheme 1 would be detrimental to the completion of the PHOBOS and BRAHMS experiments, and is not well aligned with the anticipated schedule of upgrades for STAR and PHENIX. Schemes 2 or 3 allow for a more flexible use of the available beam time and, by avoiding some setup time provide a few weeks of additional running time over the five week period. The added ability to carry out long runs in the modes listed in Table 1 is real, but certainly not decisive. 
From these exercises, one can reach the following conclusions:

- Annual runs of 27 weeks are not efficient: if one tries to run 2 species/yr, it leaves only 14 weeks for physics production/year, and introduces significant luminosity limitations. The alternative scenarios above are aimed at finding more efficient ways of handling overhead.

- In addition to integrated running weeks, the efficiency of getting to major spin physics goals depends on the frequency, duration, and timing of pp runs and of equipment installation needed to overcome present limits on both luminosity and polarization.

- All of the constant-effort scenarios considered above preserve the average of 27 weeks per year. Schemes that add flexibility in planning do have value, and should be considered, but do not solve the fundamental problem of inadequate beam time available for achieving the Five-Year Critical Goals.

In light of this last point, the group considered an incrementally improved operating scenario over the five-year period, in order to meet the requirements in Table 1. While it is impossible to make a hard-and-fast statement, an examination of Table 1, and of the Five-Year Beam Use proposals, shows that an increment of 5 weeks per year (for a total of 32 weeks per year), sustained over the 5-year period could make a major, qualitative improvement in the physics output. This would allow (for example) one "long" run of 19 weeks during each year (including setup time), plus one short, but still meaningful run of 10 weeks (again including setup time).

Table 2 shows a specific exercise carried out by the STAR collaboration, in which the 27 week beam use proposal (submitted to BNL in August 2003) is compared with an optimized Constant Effort scenario (in which runs are allowed to extend across fiscal year boundaries, but average 27 weeks per year, following Scheme 2 in Figure 1), and then with a scenario with runs of 32 weeks per year.

Comparison of Three Proposed STAR 5-Year Run Plans

\begin{tabular}{|c|c|c|c|c|c|c|}
\hline \multirow{2}{*}{$\begin{array}{c}\begin{array}{c}\text { Fiscal } \\
\text { Year }\end{array} \\
2004\end{array}$} & \multicolumn{2}{|c|}{$\begin{array}{l}27 \text { weeks/year BUP } \\
\text { (submitted } 8 / 03 \text { ) }\end{array}$} & \multicolumn{2}{|c|}{$\begin{array}{l}\text { "Optimized Constant } \\
\text { Effort" Scenario }\end{array}$} & \multicolumn{2}{|c|}{$\begin{array}{l}32 \text { weeks each year } \\
\text { run scenario }\end{array}$} \\
\hline & $\begin{array}{c}5+14 \mathrm{Au}+ \\
\mathrm{Au} 200\end{array}$ & $\begin{array}{c}5+0 p p \\
200\end{array}$ & $\begin{array}{c}5+14 \mathrm{Au}+ \\
\mathrm{Au} 200\end{array}$ & $\begin{array}{l}5+0 \mathrm{pp} \\
200\end{array}$ & $\begin{array}{c}5+14 \mathrm{Au}+ \\
\mathrm{Au} 200\end{array}$ & $\begin{array}{c}5+0 p p \\
200\end{array}$ \\
\hline \multirow{2}{*}{2005} & \multirow{2}{*}{$\begin{array}{l}5+9 \text { Au+ } \\
\text { Au Escan }\end{array}$} & \multirow{2}{*}{$\begin{array}{c}5+5 p p \\
200\end{array}$} & & & \multirow{2}{*}{$\begin{array}{c}6+8 \text { Au+ } \\
\text { Au Escan }\end{array}$} & \multirow{2}{*}{$\begin{array}{c}5+10 p p \\
200\end{array}$} \\
\hline & & & \multirow{2}{*}{$\begin{array}{l}\text { 6+11 Au+ } \\
\text { Au Escan }\end{array}$} & \multirow{2}{*}{$\begin{array}{c}5+12 p p \\
200\end{array}$} & & \\
\hline \multirow{2}{*}{2006} & \multirow{2}{*}{$\begin{array}{c}5+9 d+A u \\
200\end{array}$} & \multirow{2}{*}{$\begin{array}{c}5+5 \mathrm{pp} \\
200\end{array}$} & & & \multirow{2}{*}{$\begin{array}{c}5+8 d+A u \\
200\end{array}$} & \multirow{2}{*}{$\begin{array}{c}5+11 p p \\
200\end{array}$} \\
\hline & & & \multirow{2}{*}{$\begin{array}{c}5+9 d+A u \\
200 \\
\end{array}$} & \multirow{2}{*}{$\begin{array}{c}5+13 p p \\
200\end{array}$} & & \\
\hline \multirow{2}{*}{2007} & \multirow{2}{*}{$\begin{array}{c}5+5 \mathrm{Au}+ \\
\mathrm{Au} 200\end{array}$} & \multirow{2}{*}{$\begin{array}{c}5+9 \mathrm{pp} \\
200\end{array}$} & & & \multirow{2}{*}{$\begin{array}{c}5+10 \mathrm{Au}+ \\
\mathrm{Au} 200\end{array}$} & \multirow{2}{*}{$\begin{array}{c}5+9 \mathrm{Cu}+ \\
\mathrm{Cu} 200\end{array}$} \\
\hline & & & \multirow{3}{*}{$\begin{array}{c}5+15 \mathrm{Au}+ \\
\text { Au } 200\end{array}$} & \multirow{3}{*}{$\begin{array}{c}5+8 \mathrm{Cu}+ \\
\mathrm{Cu} 200\end{array}$} & & \\
\hline \multirow{2}{*}{2008} & \multirow{2}{*}{$\begin{array}{c}5+10 \mathrm{Au}+ \\
\mathrm{Au} 200\end{array}$} & \multirow{2}{*}{$\begin{array}{c}5+5 p p \\
500\end{array}$} & & & \multirow{2}{*}{$\begin{array}{c}5+10 \mathrm{Au}+ \\
\mathrm{Au} 200\end{array}$} & \multirow{2}{*}{$\begin{array}{c}5+9 \mathrm{pp} \\
200\end{array}$} \\
\hline & & & & & & \\
\hline $\begin{array}{l}L_{\text {max }} d t \\
\text { pp } 200\end{array}$ & \multicolumn{2}{|c|}{$76 \mathrm{pb}^{-1}$} & \multicolumn{2}{|c|}{$88 \mathrm{pb}^{-1}$} & \multicolumn{2}{|c|}{$156 \mathrm{pb}^{-1}$} \\
\hline $\begin{array}{c}\int L_{\max } d t \\
\text { post-T OF } \\
A u+A u\end{array}$ & \multicolumn{2}{|c|}{$1.4 \mathrm{nb}^{-1}$} & \multicolumn{2}{|c|}{$1.6 \mathrm{nb}^{-1}$} & \multicolumn{2}{|c|}{$2.1 \mathrm{nb}^{-1}$} \\
\hline $\begin{array}{l}\text { What's } \\
\text { missing? }\end{array}$ & \multicolumn{2}{|c|}{$\begin{array}{l}\text { Any } \mathrm{Cu}+\mathrm{Cu} 200 ; \\
2^{\text {nd }}+3^{\text {rd }} \text { long pp }\end{array}$} & \multicolumn{2}{|c|}{$\begin{array}{c}3^{\text {rd }} \text { long pp; } 2 \text { pp } \\
\text { devel. chances }\end{array}$} & \multicolumn{2}{|c|}{1 pp devel. chance } \\
\hline
\end{tabular}

Notes: 1) Luminosities are calculated in each scenario using the same model - assuming linear growth (after 5 weeks commissioning) from the previous year's maximum, at the most optimistic growth rates in the FischerRoser projections.

2) Tabulated delivered luminosities should be multiplied by 0.5 to estimate STAR recorded luminosities.

3 ) The likelihood of reaching the most optimistic pp luminosity projection increases with the number of $p p$ development chances.

Table 2. STAR comparison of 27-week/yr and 32-week/yr run plans. 
The 32 week scenario allows a striking improvement in the physics output initially proposed by STAR, increasing the physics running time by $30 \%$ and nearly doubling the amount of pp and Au-Au data obtained during the fiscal years 2005 - 2008. The Five-Year Beam Use Proposal submitted by PHENIX in August 2003, based on 27 weeks/year, contained more single-mode running than the STAR plan, driven by the desire to pay the 5-week set-up penalty only once per 27 week run. This approach increases the amount of running time relative to the STAR plan, but has the effect of delaying some key physics runs by many years, and does not allow for continuous, year-by-year development of polarized proton capability. In a subsequent planning exercise based on 32 weeks/year, similar to the STAR exercise shown in Table 2, the PHENIX collaboration found that the 32 week scenario would allow them to not only exceed the minimum requirements of Table 1 in all cases, but also to complete a second d-Au run during the 5-year period.

In order to assess the budgetary impact of such an improved scenario, we have compiled, in Table 3, the projected facility operations costs for a constant-effort ( 27 week) scenario.

\section{Table 3 appears as Appendix 4 of this document.}

Table 3 shows the actual operations costs in FY 2003, and the projected costs for running 27 weeks through FY 2008. Also shown (in parentheses) are the estimated incremental costs for additional weeks of running.

One thing to note about Table 3 is that it does not quite satisfy a constant-effort scenario (flat budgets except for inflation) in the years beyond FY 2004. This is primarily due to an anticipated one-time need for an increase in computing purchases in 2005, and an expected increase in BNL electric power costs beginning in 2006. The plan also shows an increase in detector R\&D in FY 2005 and FY 2006. Some savings must be found in order to meet the constant-effort requirement, even at the level of 27 weeks/year. BNL is committed to finding a solution to this critical issue. The table shows a \$2M savings in operations cost beginning in 2008 due to the implementation of the EBIS pre-injector, and consequent shut-down of the Tandems (see Sec. III). We also note that the "Research Equipment" lines refer to on-going and proposed upgrades to the detectors and machine that are Major Items of Equipment, costing \$2M or more, as discussed in Section III below. These are not part of the operations budget for the facility, and are regarded by DOE as competitive across the NP program. We do not include this cost category under the constant-effort constraint.

Table 3 shows a cessation of the BRAHMS and PHOBOS operations during this five-year period. Both of these groups are making plans for their continued research activities. Some members of these collaborations are expected to participate in upgrades of PHENIX and STAR, for which the constant-effort operations budgets are indicated in Table 3. Other members have indicated an interest in a new initiative to implement a detector optimized for measurements in the forward rapidity region. Our constant effort budget does not indicate any such new initiatives at this time, but the Laboratory recognizes that such ideas, on a modest scale, might be implemented as our science-driven planning evolves.

The estimated incremental cost of adding additional running weeks to a base of 27 weeks is shown in parentheses in Table 3 at about $\$ 450 \mathrm{~K} /$ week in 2004 and 2005. The estimated incremental cost for C-A operations applies only to the case of adding 1-2 weeks, on a one-time basis, such as happened in FY 2003. To increase the operations level to a new base, from 27 to 32 
weeks, requires a minimum of a 5\% (15 persons) increase in the C-A staffing level to adequately perform the operations of the facility. Thus, the total cost of adding five more weeks is estimated to be $\$ 4 \mathrm{M}$. This would be an increase of about 3\% in the operating budget for RHIC.

In summary, a number of constant-effort scheduling scenarios have been considered, all of which preserve an average of 27 cryogenic running weeks per year, with redistributions among years possible. These scenarios are budget-driven, not science-driven. BNL's RHIC Planning Group concluded that in order to successfully carry out the heavy-ion and spin programs, BNL must aim for annual running periods of 32 weeks or more. With current projections, this increment would increase the five-year physics output of RHIC by nearly a factor of two at the cost of adding 3\% per year to the FY 2005 - FY 2008 RHIC operations budget.

\section{Near-Term Upgrades for Detectors and Machine}

Important near-term upgrades for both the machine and detectors are now in the proposal phase and will require funding over the next 5 years. In our planning, these upgrades are "Major Items of Equipment" (MIE) projects funded as research equipment in addition to the RHIC operations budget, for which the Laboratory must compete for funds with other facilities in the Nuclear Physics program. The proposed upgrades for PHENIX (Si Vertex Tracker) and STAR (MRPC Time of Flight) will add essential new capability to answer questions raised by the early data at RHIC, and will be equally essential for the future measurements with high-luminosity capability of RHIC II. The EBIS pre-injector will satisfy increasing demands for reliability and stability of beams, in addition to the need for optimizing operating costs over time.

The early data from RHIC experiments have provided striking demonstration that precise and detailed measurements can be accomplished in the complex realm of high-energy collisions of heavy nuclei, and that these measurements can provide definitive experimental statements about the hot, dense matter created in the initial stages of these collisions. Each of the four RHIC detector collaborations has provided its "Decadal Plan" for detailed study of fundamental questions in quantum chromodynamics (QCD) over the next decade, exploiting this new form of matter as well as the availability of high-energy polarized proton collisions.

The Decadal Plans can be found at www.bnl.gov/henp/.

From what we know so far, it is clear that a high priority for further exploration of deconfined matter in heavy ion collisions at RHIC requires extension of the capabilities of the two large detectors, PHENIX and STAR, to identify and measure the production of high-transversemomentum particles carrying strange, charm, and bottom quarks.

Achievement of the needed improvement of detector and accelerator systems to realize these future physics goals requires a program of $R \& D$ that has gone forward for several years now. The next few years will require a step-up in the R\&D level to progress from the conceptual and proofof-principle level to the level of working prototypes and first articles. Even in the face of the severe budget guidance given for this planning exercise by DOE, BNL intends to sustain the detector and accelerator R\&D activities at the levels needed to continue making systematic progress. This policy is explicitly shown in the funding allocations of Budget Table 3. 
Supported by the R\&D work that has been carried out over the past several years, each of the two large detector collaborations has submitted a proposal for an upgrade of modest cost, using advanced technology, to implement new capability for particle detection over the next 3-5 years. These represent the initial stages of the Decadal Plans for PHENIX and STAR. These proposals will be submitted to DOE for initial funding in FY 2005.

The STAR group proposes to build a cylindrical array of 24,000 channels of time-of-flight detectors, surrounding the main Time Projection Chamber (TPC) tracking detector, with time resolution of $\sim 100$ picoseconds. This project uses the recently developed Multi-Gap Resistive Plate Chamber (MRPC) technology, that has now been well-proven in experiments at CERN and in a prototype detector that was operational in STAR during the FY 2003 RHIC run. It will allow particle identification for more than $95 \%$ of all particles produced at mid-rapidity in STAR, including multiply-strange baryons and, in conjunction with the TPC and Electromagnetic Calorimeter, will provide large samples of identified electrons from the decays of charm and bottom particles. This project is being proposed for DOE funding of $\$ 4.3 \mathrm{M}$, with an additional, in-kind contribution of $\$ 2.3 \mathrm{M}$ from China, the location where the MRPC modules will be fabricated.

The PHENIX detector collaboration proposes to build a fine-grain, high-resolution silicon tracking detector (Silicon Vertex Tracker) to provide precision measurement of heavy quark production (charm and bottom) in nucleus-nucleus, proton(deuteron)-nucleus, and polarized protonproton collisions. It will add high-resolution vertex detection to the existing powerful electron and hadron identification capability of PHENIX to provide clean selection of the (rare) decays of heavy quark states. The detector consists of two inner layers of $200 \mu \mathrm{m}$ thick silicon with $50 \times 425 \mu \mathrm{m}^{2}$ active pixels, and two outer layers of $80 \mu \mathrm{m}$ by $3.1 \mathrm{~cm}$ active strips, with a total of $\sim 1,500,000$ channels. This project is being proposed for DOE funding of $\$ 5.6 \mathrm{M}$. These funds will be supplemented by in-kind and cash contributions from the RIKEN Institute in Japan of about $\$ 3 \mathrm{M}$, during calendar years $2004-2006$.

In the meantime, with plans unfolding for long-term operation of the RHIC facility, along with increasing demands for reliability and stability of the beams, as well as the need to optimize operating costs over time, BNL has proposed an initiative for a new heavy ion pre-injector for RHIC based on the Laboratory's development of an advanced Electron Beam Ion Source (EBIS). The new pre-injector would consist of an EBIS high-charge-state ion source, a Radio Frequency Quadrupole (RFQ) accelerator, and a short Linac. Presently, one or two of BNL's 35-year old Tandem Van de Graaff accelerators are used for RHIC heavy ion pre-injection, but recent advances in the state-of-the-art in EBIS performance by more than an order of magnitude, now make it possible to meet RHIC requirements with a modern, linac-based pre-injector. The EBIS preinjector offers the following benefits:

- Improvements in reliability, setup time, and stability should lead to increased integrated luminosity in RHIC

- Reduced operating costs ( $\sim \$ 2 \mathrm{M} /$ year) and avoidance of $\sim \$ 6 \mathrm{M}$ in reliability-driven investments in the tandems that must be made if they are continued as RHIC pre-injectors

- Capability to provide ions not presently available such as uranium and, with additional enhancements, polarized ${ }^{3} \mathrm{He}$ (required for the eRHIC spin program).

The estimated cost to implement EBIS is $\$ 17.5 \mathrm{M}$, if funded over 3 years. Of this, $\$ 9.7 \mathrm{M}$ would come from existing RHIC budgets available for Accelerator Improvement Projects (AIP), and $7.8 \mathrm{M} \$$ would come from new funding provided entirely by the ONP, or supplemented by a 
contribution from NASA, because this upgrade will provide significant benefits for the NASA Space Radiation Laboratory (NSRL) facility. A pre-proposal for EBIS construction was submitted to DOE in fall 2003, requesting funding to begin in FY 2005.

A program of detector R\&D to develop the necessary technology to fully exploit highluminosity running with STAR and PHENIX, and implement measurement capability not currently possible at RHIC, was recently reviewed by an independent advisory committee appointed by BNL management. The structure and activities of this standing committee, the RHIC Detector Advisory Committee, can be found at www.bnl.gov/physics/rhic DAC.htm. The general result of the last meeting of the DAC, held at BNL on November 22, 2003, was to endorse the two detector upgrades described above, and to reiterate the importance of an aggressive detector R\&D effort to the overall RHIC program.

The Table 3 plan for detector R\&D is funded at the level of \$1M in FY 2004. As a result of this development effort, it is expected that additional upgrades may be proposed over the next few years. These include a Hadron Blind Detector (HBD) and a forward, or End Cap, Silicon Tracker (VTXe) for PHENIX, and a Micro-vertex detector (MVTX) for STAR. These are examples of projects that can enable key measurements not presently accessible, which do not initially require the full luminosity upgrade, and which are small enough that, if the technology development allows, may be funded in advance of the RHIC II project. Any such upgrades would be required to match the machine performance and physics goals envisioned for RHIC II.

\section{The Mid-Term Outlook - RHIC II}

After a decade of operations, the RHIC program as described above will reach the point where additional physics advances require integrated machine luminosities and data sets that cannot be obtained with data runs of less than 5-10 years duration using the nominal RHIC beam parameters. Further advances will require the study of extremely rare processes requiring a machine luminosity upgrade of a factor of 10 or more to restore the productivity of the facility, and corresponding detector upgrades to empower a new generation of physics advances that require these enhanced luminosity levels. The specific added physics goals are for such experiments are detailed in the decadal plans of the STAR and PHENIX collaborations.

For the RHIC facility, this upgrade of the machine and detectors is called the RHIC II Project. This project appears in the DOE's plan "Facilities for the Future of Science - a TwentyYear Outlook" announced by DOE Secretary of Energy, Spencer Abraham, on November 10, 2003. In the material below, the physics motivation for the RHIC II luminosity upgrade step is first discussed and then the specifics of this upgrade for the machine and for the PHENIX and STAR detectors is discussed. The detailed physics and instrumentation arguments for the upgrades are contained in the decadal plans referenced herein.

Current work at RHIC has shown that detailed exploration of the fundamental properties of new matter produced at RHIC can be carried out using experimental probes that carry information directly from the thermal plasma of quarks and gluons produced in these nuclear collisions. Such probes include the spectra of heavy quarks (charm and bottom) that are formed in the earliest stages of the collision, and are so massive that their dynamical properties are not lost to thermalization at the temperatures and densities that prevail in the plasma of light quarks and gluons. Another class of such probes is the measurement and classification of high-momentum jets of particles 
corresponding to energetically scattered quarks and gluons. Accurate measurements of large samples allow experiments to carry out, in effect, a tomography on the initial dense matter with "beams" of quarks and gluons.

Light quark-antiquark pair interactions measured via the spectral function of low-mass vector mesons give precise information on the massive bound states of these partons in the plasma. Heavy flavor quark-antiquark pairs, with their large quark-current masses, are less sensitive to chiral symmetry effects, and thus could carry information on the deconfinement transition. Through high statistics measurements of $\mathrm{J} / \psi, \psi$ ', $\chi_{\mathrm{c}}$ and upsilon $(1 \mathrm{~s}, 2 \mathrm{~s}, 3 \mathrm{~s})$ experiments can disentangle competing effects through comparison with lattice QCD calculations, and thus gain direct information on how the quark-antiquark potential is modified in the dense medium.

Quark-antiquark and quark-gluon interactions in a plasma should yield direct real and virtual photons that reflect the thermal history of the system. A precision measurement of this signal is a major goal of RHIC II. Furthermore, while we are currently gaining insight about light quark interactions with the medium by means of high-p T $_{\text {p }}$ probes, at RHIC II the focus will shift to the interactions of heavy quarks, which are expected to be substantially different due to the predicted "dead-cone" effect. With tagged D and B mesons, we can study how heavy quarks propagate in the medium.

These measurements, and others like them, involve extremely rare phenomena. To cite two examples from the Decadal Plans:

- The proposed measurement by PHENIX of the spectrum of Upsilon states requires a $\mathrm{Au}-\mathrm{Au}$ integrated luminosity (delivered) of $10 \mathrm{nb}^{-1}$.

- The measurement of the energy loss of heavy-quark jets in a dense medium, proposed by STAR through the measurement of $b$-anti-b jet spectra, also requires a delivered sample of $10 \mathrm{nb}^{-1}$ in Au-Au collisions.

At the current (design) luminosity, these measurements would require 160 weeks of $\mathrm{Au}-\mathrm{Au}$ running to acquire. To achieve this, along with the necessary comparison data utilizing lighter ions and varying energies, would require about a decade of facility operation in the heavy ion mode described in Sec. II above.

Such a program of measurements requires beam collision rates an order of magnitude greater than the present RHIC capability. It also requires substantial upgrades to the existing large detectors, STAR and PHENIX, to provide enhanced sensitivity to the signatures of these processes and to implement electronic readout, data acquisition, and trigger systems matched to the increased collision rates. The proposed RHIC II Project consists of major upgrades of the RHIC collider and detectors, to provide this new capability for studying extremely rare processes with high precision in high-energy nucleus-nucleus, proton-nucleus, and proton-proton collisions.

These high-luminosity heavy ion studies are essential to fully characterize the QCD matter produced at RHIC. The quark-gluon matter states produced at higher energy in the future heavy ion program at the CERN Large Hadron Collider (LHC) will likely be much more sensitive to the wee Bjorken-x behavior of the parton distribution functions and may well be fundamentally different than that produced at RHIC. The type of measurements planned for the high luminosity physics program at RHIC will not begin at the LHC until years after the initial survey phase of that program. The needed RHIC measurements will require large data samples, taken over long running 
periods with multiple beam conditions. The RHIC II facility will be uniquely suited to these physics requirements.

We now turn to a brief discussion of how BNL will achieve the enhanced machine luminosities of the RHIC II upgrades. The present rate of heavy ion collisions in RHIC is limited by intra-beam scattering, which is particularly severe with the high charge of the gold ions. The growth of the beam size due to this effect can be overcome by cooling the beams with a high intensity cold electron beam. To cool the $100 \mathrm{GeV} / \mathrm{n}$ gold beam with $10^{9}$ ions per bunch in RHIC, a $54 \mathrm{MeV}$ electron beam with an average current of about $100 \mathrm{~mA}$ is required. The high beam power of about $5 \mathrm{MW}$ of the electron beam makes it necessary to recover the beam energy by decelerating it in a superconducting linac, a technique that has been successfully demonstrated at Jefferson Lab, and is presently the focus of an R\&D effort at BNL that is funded at the level of \$2M in FY 2004. The target for Au-Au luminosity with RHIC II is $7 \times 10^{27} \mathrm{~cm}^{-2} \mathrm{sec}^{-1}$, which is approximately 40 times the present design value. Details of this plan are given in the February 2003 submission to NSAC noted below.

The PHENIX and STAR Decadal Plans identify the detector upgrades required to carry out the RHIC II physics program. These upgrades comprise:

- Precision inner tracking devices capable of directly observing charm and bottom decays

- Fast, compact, high-resolution Time Projection Chambers

- Micro-electronics for fine-grain, low-mass detectors

- Improved data acquisition and trigger techniques for very large data volumes at high rates

As noted in Section III, above, the R\&D required in order to realize these new detector capabilities has begun, and some small upgrades to the PHENIX and STAR detectors are expected to be implemented over the next 3-5 years, in advance of the RHIC II Project. The scope of the RHIC II Project includes major detector upgrades, necessary for the scientific mission of the RHIC facility, that cannot be implemented within the on-going operations and research budgets.

The ideas discussed above were captured in a short white paper that was presented to the NSAC Subcommittee on Categorizing Future Facilities, at Rutgers University on February 15, 2003. This white paper is posted on the BNL website www.bnl.gov/henp/. The purpose of the meeting was to provide a forum to present new ideas for future DOE nuclear physics facilities. The NSAC sub-panel was charged to assign each of the presented ideas to one of three DOE-provided categories ("absolutely central to progress in the field"; "important"; "not yet ready for consideration"). BNL submitted the RHIC II and eRHIC concepts to this workshop (as a single document/presentation at the direction of DOE) and was pleased to learn that both concepts were rated "absolutely central to the field" by NSAC. This NSAC rating was sustained when the DOE's plan for future facilities, "Facilities for the Future of Science-a Twenty-Year Outlook" announced by DOE Secretary of Energy, Spencer Abraham, on November 10, 2003. Both RHIC II and eRHIC were included on the future facilities list.

The cost of RHIC II was estimated in the above-referenced BNL "RHIC II/eRHIC" white paper at $\$ 153 \mathrm{M}$ FY03 dollars. The project will be ready to start construction around 2008, a time scale that is consistent with that indicated in the DOE facilities plan. As presently envisioned, the RHIC II upgrades will be implemented without requiring a lengthy shutdown of the facility, allowing the heavy ion and spin programs to proceed without interruption. 
The scientific importance of the RHIC II upgrades was also acknowledged in the most recent NSAC Long Range Plan, published in April 2002, which states: "An upgrade program such as the RHIC II initiative will allow in-depth pursuit of the most promising observables characterizing the deconfined state." The next Long Range Plan process is expected to begin in 2005. If RHIC II is to be realized on the timescale foreseen here, it will have to be thoroughly discussed as a part of this planning process. Thus, it will be important over the coming year to fully develop the specific scope of the detector upgrades to be associated with this project.

We should note that some new ideas for future detectors have been brought forward that are not related to the specific plans of existing collaborations (see, for example the agenda of the Open Meeting for RHIC Planning, given in Appendix 2, and talks on the indicated BNL web site by F. Videbaek and J. Harris). Flemming Videbaek of BNL presented ideas for a new forward detector, and John Harris of Yale presented a design concept for a new large solid-angle detector for heavy ion and spin measurements.

\section{The Long-Term Outlook - eRHIC}

The next step in physics research capability for the RHIC facility after the luminosity upgrade provided by RHIC II is to add an electron ring of $10 \mathrm{GeV}$ energy capability (and tunable over the range 5-10 GeV) that collides polarized electron beams with beams in one of the existing RHIC heavy ion/polarized proton rings, together with a new detector that is optimized to study the collisions of electrons with heavy ions and polarized protons. The realization of this new physics capability will be provided in the eRHIC Construction Project. This project has been carefully scoped to minimize the interruption of the existing RHIC program during the eRHIC construction period. Even after eRHIC begins to operate, it will be possible to continue running the RHIC II program in four of the six original colliding beam interaction points.

In the sections below, we first identify the new physics that can be achieved with the collisions of very high energy electrons and ions and then go on to describe the machine parameters that will enable these collisions to take place. We also briefly describe the community that will benefit from the eRHIC facility and the status of approval of the eRHIC concept in the DOE future plans. The physics, accelerator and detector concepts that are central to eRHIC are discussed in detail in the "Electron-Ion Collider White Paper", Feb. 2002, which can be found on the web at www.bnl.gov/henp/.

With strong roots in the heavy ion program, the spin program, and the $p(d)-A$ measurements in RHIC and RHIC II, eRHIC will probe the fundamental quark and gluon structure of strongly interacting matter with high precision. The new scientific opportunities include:

- $\quad$ Partonic matter under extreme conditions: eRHIC, running in eP and eA modes, would allow unprecedented measurements of the proton structure and its evolution into nuclear structure. In particular, the structure functions $\mathrm{F}_{2}, \mathrm{~F}_{\mathrm{L}}$, and $\mathrm{xF}_{3}$, will be measured in new kinematical ranges, and with unmatched precision. The structure function measurements on protons will allow the study of the transition region from a partonic to a hadronic description of strongly interacting matter, and will allow stringent tests of the standard perturbative QCD tools. The same series of measurements with nuclei will allow the search for the saturated gluon state the Color Glass Condensate. The extracted parton densities will be required for a full understanding of the heavy ion collisions and the quark gluon plasma. 
- $\quad$ The black disk limit: Diffractive scattering was observed in over $10 \%$ of all DIS events at HERA. eRHIC operation with nuclei should allow the observation of a far greater fraction of diffractive events, approaching the quantum mechanical limit of 50\%. The detailed diffractive data will provide a stringent test on our understanding of the strong interactions.

- $\quad$ Three Dimensional Mapping of Strong Matter: The study of exclusive reactions, such as the production of vector mesons or real photons, will allow the mapping of strongly interacting matter in nucleons and nuclei. These data are sure to bring a great leap forward in our understanding of how nuclear matter is formed, and will be critical in the search for the Color Glass Condensate.

- $\quad$ Radiation Patterns in Strong Interactions: The study of the fundamental radiation patterns in strong interactions, which lead to the small-x structure of nucleons, will be studied by studying jet and particle production over a large rapidity range. The eRHIC accelerator, supplemented with an optimized detector, would allow a greatly enhanced look at the correlations and fluctuations in gluon and quark radiation at the heart of all matter.

- $\quad$ Hadronization in nucleons and nuclei: The evolution of colored quarks and gluons struck by the virtual photon in deep inelastic scattering into observed colorless hadrons is one of the clearest manifestations of confinement. eRHIC will make it possible to observe the complete array of decay products in deeply inelastic scattering of electrons from the nucleon and nuclei.

- Quark and gluon distributions in the nucleon: eRHIC offers a unique capability for measuring 'flavor-tagged' structure functions by providing access to a wide range of final states. Quark and gluon distributions in nucleons, nuclei, and possibly even mesons can be mapped in a flavor tagged mode.

- Spin structure of the nucleon: eRHIC, operating at the highest center-of-mass energy, will provide data on the proton's spin-dependent structure functions at lower $x$ than possible in any previous experiment. This information, along with that from scaling violations, will likely be indispensable for obtaining definitive answers about the quark and gluon spin contributions to the proton spin. Measurements of the spin structure functions $g_{1}$ of proton and neutron (possibly using polarized ${ }^{3} \mathrm{He}$ beams) will allow a precision test of the Bjorken sum rule. At highest momentum transfer, parity-violation in electroweak structure functions will be accessible. In addition, eRHIC, with its variable electron and proton beam energies, will also be ideal for exploring the nucleon spin structure in the valence region.

When eRHIC is inaugurated, RHIC will be providing high luminosity, electron-cooled ion beams and high luminosity, highly polarized proton beams. The present design of an electron-ion collider, based on the existing RHIC machine, requires the construction of a $10-\mathrm{GeV}$ electron accelerator ring with a beam-energy tuning range of 5-10 GeV, that will have $1 / 3$ of the RHIC circumference and collides with the ion beam in one of the existing RHIC experimental areas. The electron beam is produced by polarized electron source and then accelerated to collision energies by full energy linear accelerator. In order to minimize electron linac size and cost, beam recirculation is used to pass the electron beam twice through the same linac accelerator sections. Positron beam also can be accelerated and used, but the polarization must then be acquired in the ring itself by the slower synchrotron radiation process. 
The electron ring consists of two arcs with regular FODO structure and two straight sections: one includes the interaction region and the other injection and $\mathrm{rf}$ systems. The polarization setup for the electron beam also includes a pair of solenoidal spin rotators around the interaction region to produce longitudinal polarization. The RHIC ion rings already have dedicated magnet insertions, Siberian Snakes and spin rotators, in order to provide high energy polarized proton beam for the collisions.

Electron cooling of the Au beam, which will be provided in the RHIC rings by the RHIC II project, is necessary to provide the projected luminosity for electron-gold collisions. With an extensive R\&D effort planned, the ultimate goal for eRHIC is to achieve an average luminosity of $1 \times 10^{33} \mathrm{~cm}^{-2} \mathrm{sec}^{-1}$ for e-p and $1 \times 10^{31} \mathrm{~cm}^{-2} \mathrm{sec}^{-1}$ for e-Au collisions.

The main beam parameters in the present design for high-energy e-p and e-Au collisions are shown in Table 4 below.

\begin{tabular}{|l|c|c||c|c|}
\hline \multirow{2}{*}{} & \multicolumn{2}{|c||}{ e-p collisions } & \multicolumn{2}{c|}{ e-Au collisions } \\
\cline { 2 - 5 } & $\mathrm{p}$ & $\mathrm{e}$ & $\mathrm{Au}$ & $\mathrm{e}$ \\
\hline Circumference [m] & 3833 & 1278 & 3833 & 1278 \\
\hline Energy [GeV] & 250 & 10 & $100 / \mathrm{u}$ & 10 \\
\hline Number of bunches & 360 & 120 & 360 & 120 \\
\hline Bunch population $\left[10^{11}\right]$ & 1 & 1 & 0.01 & 1 \\
\hline Beam current [A] & 0.45 & 0.45 & 0.36 & 0.45 \\
\hline Rms emittance [10-9 $\mathrm{m}], \mathrm{x} / \mathrm{y}$ & $9.5 / 9.5$ & $53 / 9.5$ & $9.5 / 9.5$ & $53 / 7.5$ \\
\hline Beta function at IP [cm], x/y & $108 / 27$ & $19 / 27$ & $108 / 27$ & $19 / 34$ \\
\hline Beam size at IP [mm], x/y & \multicolumn{2}{|c|}{$0.1-0.05$} & \multicolumn{2}{c|}{$0.1-0.05$} \\
\hline Beam-beam parameter, x/y & $0.007 / 0.003$ & $0.029 / 0.08$ & $0.007 / 0.003$ & $0.022 / 0.08$ \\
\hline Luminosity, [1032 cm-2 s-1] & \multicolumn{3}{|c|}{4.4} \\
\hline
\end{tabular}

\section{Table 4. Conceptual design beam parameters for the eRHIC Facility.}

A second possible design for the electron machine, that brings polarized electron beam into the collision point directly from a superconducting energy recovery linac, is also under consideration for eRHIC.

The preliminary cost estimate for eRHIC, $\$ 511 \mathrm{M}$ FY 03 dollars, was provided in the "RHIC II/eRHIC" white paper presented to the NSAC future facilities sub-panel in February 2003. This cost estimate includes the addition of a new detector optimized for e-A and polarized e-p collisions. Construction could start around 2010 if driven only by technical factors; a construction start later in the next decade is foreseen in the DOE's 20-year facility plan.

A significantly expanded scientific community will be served by the facility when eRHIC operations commence. Segments of the QCD and hadronic physics communities in the US (Fermilab, Jlab, SLAC) and Europe (DESY/HERA/HERMES, CERN/Compass) are expected to 
participate in the research program and are now getting involved in the formulation of the eRHIC physics program at various levels, including IR and detector design. Further, the MIT/Bates Laboratory has concluded a collaborative agreement with BNL on the eRHIC project and is involved deeply in the design of the electron ring. This involvement will continue through the eRHIC construction project period. 


\section{Appendix 1}

\section{RHIC Planning Group}

Convenors: T. Kirk, T. Ludlam

\begin{tabular}{|l|l|l|l|}
\hline PHENIX & STAR & PHOBOS & BRAHMS \\
G. Bunce & W. Christie & M. Baker \\
A. Drees & T. Hallman & $\begin{array}{l}\text { G. Roland } \\
\text { E. O'Brien }\end{array}$ & P. Steinberg \\
W. Zajc & $\begin{array}{l}\text { R. Majka } \\
\text { S. Vigdor }\end{array}$ & & \\
\hline $\begin{array}{l}\text { Accelerator } \\
\text { J. Alessi }\end{array}$ & Theory & Computing \\
I. Ben Zvi & D. Kharzeev & B. Gibbard & PAC/DAC Invited \\
W. Fischer & W. Vogelsang & T. Throwe & R. Betts \\
P. Pile & & & P. Jacobs \\
V. Ptitsyn & & & S.-Y. Lee \\
T. Roser & & & J. Nagle \\
\hline
\end{tabular}

Ex Officio: S. Aronson, D. Lowenstein, P. Paul 


\section{Appendix 2 \\ Open Meeting on RHIC Planning - Brookhaven National Lab \\ December 3-4, 2003 - www.bnl.gov/henp/}

\begin{tabular}{|c|c|c|}
\hline \multicolumn{3}{|c|}{ Wednesday Dec. 3} \\
\hline Chair & S. Aronson & \\
\hline $9: 00$ & Welcome & T. Kirk \\
\hline $9: 15$ & Presentation and discussion of White Paper & T. Ludlam \\
\hline $10: 40$ & Break & \\
\hline $11: 00$ & $\begin{array}{l}\text { QGP Physics: outlook; major questions and } \\
\text { experimental issues for the long term }\end{array}$ & S. Milov \\
\hline $\begin{array}{l}11: 40 \\
12: 20\end{array}$ & $\begin{array}{c}\text { Spin Physics: the baseline goals and beyond } \\
\text { Lunch }\end{array}$ & S. Vigdor \\
\hline Chair & R. Betts & \\
\hline $2: 00$ & $\frac{\text { eRHIC/pA/low-x Physics: (including }}{\text { forwardphysics in RHIC II) }}$ & R. Venugopalan \\
\hline $2: 40$ & Near-term machine performance & W. Fischer \\
\hline 3:05 & PHOBOS near-term plans & G. Roland \\
\hline $3: 30$ & PHENIX Decadal Plan & W. Zajc \\
\hline $4: 15$ & Break & \\
\hline $4: 30$ & STAR Decadal Plan & T. Hallman \\
\hline $5: 15$ & Forward Physics initiative & F. Videbaek \\
\hline $5: 40$ & Adjourn & \\
\hline 6:00 & Reception and dinner & \\
\hline
\end{tabular}

Thursday Dec. 4

\begin{tabular}{|c|c|c|}
\hline Chair & P. Jacobs & \\
\hline $8: 30$ & EBIS status and plans & J. Alessi \\
\hline 9:00 & RHIC II machine plans & T. Roser \\
\hline $9: 30$ & STAR R\&D and upgrade plan & R. Majka \\
\hline 10:10 & Break & \\
\hline $10: 30$ & PHENIX R\&D and upgrade plan & A. Drees \\
\hline $11: 10$ & $\begin{array}{l}\text { A new detector for in-depth study of QGP and } \\
\text { spin physics at RHIC II }\end{array}$ & J. Harris \\
\hline $11: 40$ & eRHIC Machine Plans & V. Ptitsin \\
\hline $12: 10$ & Lunch & \\
\hline Chair & P. Bond & \\
\hline $1: 30$ & IR design for eRHIC & C. Montag \\
\hline $1: 45$ & eRHIC detector and experimental program & A. Deshpande \\
\hline $2: 25$ & Future DIS experiments at HERA III/eRHIC & A. Caldwell \\
\hline $3: 10$ & Break & \\
\hline $3: 30$ & $\begin{array}{l}\text { Comments from the floor } \\
\text { Elastic Scatt }(\text { Gurvn } 10 \mathrm{~min} \text { ) }\end{array}$ & W Gurvn \\
\hline 4:00 & Final discussion of white paper & S. Aronson \\
\hline $5: 30$ & Adjourn & \\
\hline
\end{tabular}




\section{Appendix 3 - Web References}

The documents referenced in this paper have been assembled on the BNL website, http:/www.bnl.gov/henp/ . They are listed by subject here to facilitate easy location on that site. Within the references noted here, there are further URLs to allow investigation of details as needed.

Five-Year Beam Request Proposals of BRAHMS, PHENIX, PHOBOS and STAR

Decadal Plans of BRAHMS, PHENIX, PHOBOS and STAR

Open Meeting on RHIC Planning Brookhaven National Laboratory, December 3-4, 2003

BNL HENP Program Advisory Committee (PAC) Documents on Future Planning

BNL Detector Advisory Committee Document on the RHIC R\&D Program

BNL White Paper Presented to the NSAC Future Facilities Workshop of February 15, 2003

The Electron Ion Collider White Paper, February 2002 
Appendix 4 - Budget Table for FY 2004 - FY 2008

Table 3 RHIC Planning: Constant-Effort Budget case (27 wks/yr)

FY 2003 entries are in actual dollars; subsequent years are in FY 2004 dollars 12/29/03 TL

\begin{tabular}{|c|c|c|c|c|c|c|}
\hline Fiscal Year & 2003 & 2004 & 2005 & 2006 & 2007 & 2008 \\
\hline $\begin{array}{l}\text { PHENIX } \\
\text { Ops Costs } \\
\text { R\&D } \\
\text { Ops Equip. } \\
\text { Res. Equ. } \\
\end{array}$ & $\begin{array}{l}\$ 6.0 \mathrm{M}(24 \mathrm{~K} / \mathrm{wk}) \\
\$ 0.12 \mathrm{M} \\
\$ 0.5 \mathrm{M}\end{array}$ & $\begin{array}{l}5.85 \mathrm{M}(27 \mathrm{~K} / \mathrm{wk}) \\
0.5 \mathrm{M} \\
0.89 \mathrm{M}\end{array}$ & $\begin{array}{l}5.85 \mathrm{M}(42 \mathrm{~K} / \mathrm{wk}) \\
0.95 \mathrm{M} \\
0.89 \mathrm{M} \\
2.5 \mathrm{M} \mathrm{VTXb} \\
\end{array}$ & $\begin{array}{l}5.85 \mathrm{M}(42 \mathrm{~K} / \mathrm{wk}) \\
0.6 \mathrm{M} \\
0.89 \mathrm{M} \\
2.5 \mathrm{M} \text { VTXb } \\
\end{array}$ & $\begin{array}{l}5.85 \mathrm{M}(42 \mathrm{~K} / \mathrm{wk}) \\
0.3 \mathrm{M} \\
0.89 \mathrm{M} \\
4.2 \mathrm{M} \mathrm{VTXb/e} \\
\end{array}$ & $\begin{array}{l}5.85 \mathrm{M}(42 \mathrm{~K} / \mathrm{wk}) \\
-- \\
0.89 \mathrm{M} \\
4.5 \mathrm{M} \mathrm{VTXe}\end{array}$ \\
\hline $\begin{array}{l}\text { STAR } \\
\text { Ops Costs } \\
\text { R\&D } \\
\text { Ops Equip. } \\
\text { Res. Equ. }\end{array}$ & $\begin{array}{l}\$ 5.9 \mathrm{M}(38 \mathrm{~K} / \mathrm{wk}) \\
\$ 0.12 \mathrm{M} \\
\$ 0.49 \mathrm{M} \\
\$ 3.0 \mathrm{M} \mathrm{BEMC} \\
{[\$ 1.5 \mathrm{M} \mathrm{EEMC}]}\end{array}$ & $\begin{array}{l}5.75 \mathrm{M}(40 \mathrm{~K} / \mathrm{wk}) \\
0.5 \mathrm{M} \\
0.99 \mathrm{M} \\
1.95 \mathrm{M} \mathrm{BEMC}\end{array}$ & $\begin{array}{l}5.75 \mathrm{M}(40 \mathrm{~K} / \mathrm{wk}) \\
1.0 \mathrm{M} \\
0.99 \mathrm{M} \\
2.0 \mathrm{M} \mathrm{TOF}\end{array}$ & $\begin{array}{l}5.75 \mathrm{M}(40 \mathrm{~K} / \mathrm{wk}) \\
1.28 \mathrm{M} \\
0.99 \mathrm{M} \\
4.0 \mathrm{M} \text { TOF, MVTX }\end{array}$ & $\begin{array}{l}5.75 \mathrm{M}(40 \mathrm{~K} / \mathrm{wk}) \\
0.3 \mathrm{M} \\
0.99 \mathrm{M} \\
4.5 \mathrm{M} \mathrm{MVTX}\end{array}$ & $\begin{array}{l}5.75 \mathrm{M}(40 \mathrm{~K} / \mathrm{wk}) \\
--- \\
0.99 \mathrm{M} \\
\text { 3.0M MVTX }\end{array}$ \\
\hline $\begin{array}{c}\text { PHOBOS } \\
\text { Ops Costs } \\
\text { Ops Equ. }\end{array}$ & $\$ 0.86 \mathrm{M}(10 \mathrm{~K} / \mathrm{wk})$ & $\begin{array}{l}0.75 \mathrm{M}(10 \mathrm{~K} / \mathrm{wk}) \\
0.185 \mathrm{M}\end{array}$ & $\begin{array}{l}0.75 \mathrm{M}(10 \mathrm{~K} / \mathrm{wk}) \\
0.185 \mathrm{M}\end{array}$ & $\begin{array}{l}0.75 \mathrm{M}(10 \mathrm{~K} / \mathrm{wk}) \\
0.185 \mathrm{M}\end{array}$ & $\begin{array}{l}.75 \mathrm{M}(10 \mathrm{~K} / \mathrm{wk}) \\
----\end{array}$ & ------------- \\
\hline $\begin{array}{c}\text { BRAHMS } \\
\text { Ops Costs } \\
\text { Ops Equ. }\end{array}$ & $\$ 0.78 \mathrm{M}(10 \mathrm{~K} / \mathrm{wk})$ & $\begin{array}{l}0.7 \mathrm{M}(10 \mathrm{~K} / \mathrm{wk}) \\
0.11 \mathrm{M}\end{array}$ & $\begin{array}{l}0.7 \mathrm{M}(10 \mathrm{~K} / \mathrm{wk}) \\
0.1 \mathrm{M}\end{array}$ & $\begin{array}{l}0.7 \mathrm{M}(10 \mathrm{~K} / \mathrm{wk}) \\
0.1 \mathrm{M}\end{array}$ & ----------- & -------------- \\
\hline $\begin{array}{l}\text { RCF } \\
\text { Ops Costs } \\
\text { Ops Equ. }\end{array}$ & $\begin{array}{l}\$ 5.18 \mathrm{M} \\
\$ 2.0 \mathrm{M} \\
\end{array}$ & $\begin{array}{l}5.31 \mathrm{M} \\
2.0 \mathrm{M} \\
\end{array}$ & $\begin{array}{l}5.6 \mathrm{M} \\
3.4 \mathrm{M} \\
\end{array}$ & $\begin{array}{l}5.6 \mathrm{M} \\
2.0 \mathrm{M} \\
\end{array}$ & $\begin{array}{l}5.6 \mathrm{M} \\
2.0 \mathrm{M} \\
\end{array}$ & $\begin{array}{l}5.6 \mathrm{M} \\
2.0 \mathrm{M} \\
\end{array}$ \\
\hline $\begin{array}{l}\text { C-AD } \\
\text { Ops Costs } \\
\text { R\&D } \\
\text { Ops Equip. } \\
\text { Res. Equ. } \\
\end{array}$ & $\begin{array}{l}\$ 90.3 \mathrm{M}(350 \mathrm{~K} / \mathrm{w}) \\
\$ 0.9 \mathrm{M} \\
\$ 4.4 \mathrm{M} \\
---\end{array}$ & $\begin{array}{l}90.7(350 \mathrm{~K} / \mathrm{wk}) \\
2.0 \mathrm{M} \\
3.9 \mathrm{M} \\
---\end{array}$ & $\begin{array}{l}90.9 \mathrm{M}(350 \mathrm{~K} / \mathrm{wk}) \\
2.0 \mathrm{M} \\
3.8 \mathrm{M} \\
2.5 \mathrm{M} \mathrm{EBIS} \\
\end{array}$ & $\begin{array}{l}94.9 \mathrm{M}(500 \mathrm{~K} / \mathrm{w}) \\
2.0 \mathrm{M} \\
3.8 \mathrm{M} \\
2.5 \mathrm{M} \text { EBIS }\end{array}$ & $\begin{array}{l}94.9 \mathrm{M}(500 \mathrm{~K}) \\
2.0 \mathrm{M} \\
3.8 \mathrm{M} \\
2.5 \mathrm{M} \mathrm{EBIS}\end{array}$ & $\begin{array}{l}92.8 \mathrm{M}(500 \mathrm{~K}) \\
2.0 \mathrm{M} \\
3.8 \mathrm{M} \\
---\end{array}$ \\
\hline Users/CAP & $\$ 0.86 \mathrm{M}$ & $0.90 \mathrm{M}$ & $0.90 \mathrm{M}$ & $0.90 \mathrm{M}$ & $0.90 \mathrm{M}$ & $0.90 \mathrm{M}$ \\
\hline $\begin{array}{l}\text { Totals } \\
\text { Ops costs } \\
\text { R\&D } \\
\text { Ops Equip. } \\
\text { Ops Total } \\
\text { Res. Equ. } \\
\end{array}$ & $\begin{array}{ll}\text { \$109.8M }(432 \mathrm{~K}) \\
\text { \$1.1M } & \\
\text { \$7.4M } & \\
& \\
\text { Actual: } & \text { \$118.4M } \\
\text { \$3.0M } & \\
\end{array}$ & $\begin{array}{ll}\text { \$110.1M }(437 \mathrm{~K}) \\
\text { \$3.0M } & \\
\text { \$8.0M } & \\
& \\
\text { Pres: } & \text { \$121.0M } \\
\text { \$1.95M } & \\
\end{array}$ & $\begin{array}{ll}\$ 110.4 \mathrm{M}(452 \mathrm{~K}) \\
\$ 4.0 \mathrm{M} & \\
\$ 9.4 \mathrm{M} & \\
& \\
& \$ 123.8 \mathrm{M} \\
\$ 7.0 \mathrm{M} & \\
\end{array}$ & $\begin{array}{l}\text { \$114.4M }(602 \mathrm{~K}) \\
\$ 3.9 \mathrm{M} \\
\$ 8.0 \mathrm{M} \\
\\
\$ 99.1 \mathrm{M}\end{array}$ & $\begin{array}{l}\text { \$113.8M (592K) } \\
\$ 2.6 \mathrm{M} \\
\$ 7.9 \mathrm{M} \\
\\
\end{array}$ & $\begin{array}{ll}\$ 110.9 \mathrm{M}(582 \mathrm{~K}) \\
\$ 2.0 \mathrm{M} \\
\$ 7.7 \mathrm{M} \\
& \\
& \\
\$ 7.5 \mathrm{M} & \\
\end{array}$ \\
\hline
\end{tabular}

